\title{
Postikale Hyper-CK-Ämie und antiepileptikainduzierte Myopathie bzw. Rhabdomyolyse nicht vergessen!
}

\section{Leserbrief zu}

Sieb JP (2021) Erhöhte Kreatinkinase-Serumaktivität (Hyper-CK-Ämie). DGNeurologie 4: 31-34. https://doi.org/10.1007/s42451-020-00287-2

\section{Erwiderung}

Sieb JP (2021) CK-Serumaktivität und Epilepsie. https://doi.org/10.1007/ s42451-021-00325-7

Die sehr schöne Übersicht von Sieb (SOP/Algorithmus) in Heft 1/2021 zu den möglichen Ursachen einer erhöhen Kreatinkinaseserumaktivität [1] bedarf einer praxisrelevanten Ergänzung. Nach bilateralen tonisch-klonischen bzw. generalisierten epileptischen (Grand-Mal)-Anfällen - auch nach fiebergebundenen Anfällen (sog. Fieberkrämpfen) von Kleinkindern - kann es mit einer Latenz von bis zu einigen Tagen $\mathrm{zu}$ einem passageren starken Anstieg der CK auf Werte bis zu über 60.000 IU kommen [2-10]. Wenn diese Möglichkeit außer Acht gelassen wird, besteht die Gefahr einer überflüssigen Diagnostik. Erfahrene Epileptologen benutzen dies sogar zur Bewertung kürzlicher Anfallsereignisse; zwar ist die Sensitivität nach konvulsiven Anfällen mit $10-20 \%$ recht bescheiden, die Spezifität bei einer Erhöhung hinsichtlich der Abgrenzung nichtepileptischer Anfälle aber hoch [8-10].

Darüber hinaus sollten auch die genannten myotoxischen Medikamente (Statine, Chloroquin) durch Antiepileptika ergänzt werden. Auch wenn dies selten ist, gibt es für fast alle gängigen Antiepileptika Mitteilungen einer induzierten Myopathie bzw. Rhabdomyolyse [11-21].

Literatur

1. Sieb JP (2021) Erhöhte Kreatinkinase-Serumaktivität (Hyper-CK-Ämie). DGNeurologie 4:31-34

2. Belton NR, Backus RE, Millichap JG (1967) Serum creatine phosphokinase activity in epilepsy. Clinical and experimental studies. Neurology 17:1073-1076

3. Chesson AL, Kasarskis EJ, Small VW (1983) Postictal elevation of serum creatine kinase levels. Arch Neurol 40:315-317

4. Wyllie E, Lueders H, Pippenger C, VanLente F (1985) Postictal serum creatine kinase in the diagnosis of seizure disorders. Arch Neurol 42:123-126

DGNeurologie 2021 · 4 (3): 231

https://doi.org/10.1007/s42451-021-00324-8

Angenommen: 15. Februar 2021

๑) Springer Medizin Verlag GmbH, ein Teil von Springer Nature 2021
5. Lahat E, Eshel G, Heyman E et al (1989) Elevated serum creatine kinase following febrile seizures. Clin Pediatr 28:449-451

6. Besser R, Krämer G (1990) Verzögerte CK-Erhöhung nach Grand mal-Anfällen. Akt Neurol 17:117-119

7. Neufeld MY, Treves TA, Chistik V, Korczyn AD (1997) Sequential serum creatine kinase determination differentiates vaso-vagal syncope from generalized tonic-clonic seizures. Acta Neurol Scand 95:137-139

8. Willert C, Spitzer C, Kusserow S, Runge U (2004) Serum neuron-specific enolase, prolactin, and creatine kinase after epileptic and psychogenic non-epileptic seizures. Acta Neurol Scand 109:318-323

9. Holtkamp M, Othman J, Buchheim K, Meierkord H (2006) Diagnosis of psychogenic nonepileptic status epilepticus in the emergency setting. Neurology 2006(66):1727-1729

10. Petramfar P, Yaghoobi E, Nemati R, Asadi-Pooya AA (2009) Serum creatine phosphokinase is helpful in distinguishing generalized tonic-clonic seizures from psychogenic nonepileptic seizures and vagovasal syncope. Epilepsy Behav 15:330-332

11. Engel JN, Mellul VG, Goodman DB (1986) Phenytoin hypersensitivity: a case of severe acute rhabdomyolysis. Am J Med 81:928-930

12. Kumar N, Khwaja GA, Gupta M, Sharma S (1993) Antiepileptic drug induced osteomalacic myopathy with hyperparathyroidism and nephrolithiasis. J Assoc Physicians India 41:748-749

13. Barclay CL, McLean M, Hagen Net al (1992) Severe phenytoin hypersensitivity with myopathy: a case report. Neurology 42:2303

14. Kottlors M, Jaksch M, Ketelsen UP et al (2001) Valproic acid triggers acute rhabdomyolysis in a patient with carnitine palmitoyltransferase type Il deficiency. Neuromuscul Disord 11:757-759

15. Schwartz MD, Geller RJ (2007) Seizures and altered mental status after lamotrigine overdose. Ther Drug Monit 29:843-844

16. Tuccori M, Lombardo G, Lapi F et al (2007) Gabapentin-induced severe myopathy. Ann Pharmacother 41:1301-1305

17. Huang LY, Lin CM, Chiou CC et al (2012) Rhabdomyolysis as a potential complication of carbamazepine-induced toxic epidermal necrolysis. Clin Biochem 45:1531-1532

18. Gunathilake R, Boyce LE, Knight AT (2013) Pregabalin-associated rhabdomyolysis. Med J Aust 199:624-625

19. Akiyama H, Haga Y, Sasaki N et al (2014) A case of rhabdomyolysis in which levetiracetam was suspected as the cause. Epilepsy Behav Case Rep 2:152-155

20. Wang X, Zhou S (2016) Rhabdomyolysis induced by antiepileptic drugs: characteristics, treatment and prognosis. Saf, Bd. 15. Expert, Opin Drug, S 357-365 21. Di Lorenzo R, Li Y (2017) Rhabdomyolysis associated with levetiracetam administration. Muscle Nerve 56:E1-E2

\section{Korrespondenzadresse}

\section{Dr. med. Günter Krämer}

Neurozentrum Bellevue

Theaterstr. 8, 8001 Zürich, Schweiz

g.kraemer@epilepsie-med.de

Interessenkonflikt. G. Krämer gibt an, dass kein Interessenkonflikt besteht. 